\title{
The Plasticity of Some Fittable Surfaces on a Given Quadruple of Points in the Three-Dimensional Euclidean Space
}

\author{
A.N. Zachos \\ University of Patras, Department of Mathematics \\ GR-26500 Rion, Greece \\ E-mail: azachos@gmail.com
}

Received September 1, 2013, revised April 4, 2014

\begin{abstract}
We construct a two-dimensional sphere in the three-dimensional Euclidean space which intersects a circular cylinder in three given points and the corresponding weighted Fermat-Torricelli point for a geodesic triangle such that these three points and the corresponding weighted FermatTorricelli point remain the same on the sphere for a different triad of weights which correspond to the vertices on the surface of the sphere. We derive a circular cone which passes from the same points that a circular cylinder passes. By applying the inverse weighted Fermat-Torricelli problem for different weights, we obtain the plasticity equations which provide the new weights of the weighted Fermat-Torricelli point for fixed geodesic triangles on the surface of a fittable sphere and a fittable circular cone with respect to the given quadruple of points on a circular cylinder, which inherits the curvature of the corresponding fittable surfaces.
\end{abstract}

Key words: weighted Fermat-Torricelli point, sphere, circular cylinder, circular cone, fittable surfaces.

Mathematics Subject Classification 2010: 51E12, 52A10, 52A55, 51E10.

\section{Introduction}

The weighted Fermat-Torricelli problem states that:

Given are three points $A_{1}, A_{2}, A_{3}$ in the Euclidean plane, three positive real numbers $w_{i}$ (weight) which correspond to the vertex $A_{i}$, find a point $X$ in the Euclidean plane that minimizes the sum of the weighted Euclidean distances $f(X)=w_{1}\left\|A_{1} X\right\|+w_{2}\left\|A_{2} X\right\|+w_{3}\left\|A_{3} X\right\|$.

The solution of the weighted Fermat-Torricelli problem is named as the weighted Fermat-Torricelli point $F$. E. Torricelli was the first to discover the 
isogonal property (or $120^{\circ}$ property) of the weighted Fermat-Torricelli point $\angle A_{1} F A_{2}=\angle A_{2} F A_{3}=\angle A_{3} F A_{1}=120^{\circ}$ for equal weights. B. Cavalieri was the first who stated that if at most one angle $\angle A_{i} A_{j} A_{k} \geq 120^{\circ}$, then $F=A_{j}$ for $w_{1}=w_{2}=w_{3}, i, j, k=1,2,3, i \neq j \neq k$ (see $[2,4]$ ).

The isogonal property of the equally weighted Fermat-Torricelli point holds in Riemmanian manifolds ([3]) and in an Alexandrov surface of the bounded curvature ([5], in the surface of polyhedra).

We introduce a problem of the (curvature) plasticity of a surface which passes from four given points in $\mathbb{R}^{3}$ :

Problem 1 (Problem of plasticity of fittable surfaces in $\mathbb{R}^{3}$ ). Suppose that $F$ is the corresponding weighted Fermat-Torricelli point of a geodesic triangle $\triangle A_{1} A_{2} A_{3}$ on a $C^{2}$ complete surface $M$ with weights $w_{1}, w_{2}$, and $w_{3}$. Find a fittable Alexandrov surface $M^{\prime}$ of the bounded curvature which passes from $A_{1}$, $A_{2}, A_{3}$, and $F$ such that $F$ is the corresponding weighted Fermat-Torricelli point of $\triangle A_{1} A_{2} A_{3}$ on $M^{\prime}$ with weights $w_{1}^{\prime}$, we, and $w_{3}^{\prime}$.

In this paper, we apply the weighted Fermat-Torricelli problem for geodesic triangles on certain surfaces in the three- dimensional Euclidean space, the inverse weighted Fermat-Torricelli problem, in order to derive the equations which allow us to compute the weights corresponding to the fittable surfaces for three fixed points and a fixed fourth point (weighted Fermat-Torricelli point) located at the interior of the geodesic triangle for the case of a two-dimensional sphere in the three-dimensional Euclidean space which intersects a circular cylinder in three given points and the corresponding weighted Fermat-Torricelli point for a geodesic triangle and a fittable circular cone which passes from the same points that a circular cylinder passes.

\section{Plasticity of a Sphere and Circular Cone with Respect to a Circular Cylinder in the Three-Dimensional Euclidean Space}

Let $\triangle\left(A_{1} A_{2} A_{3}\right)_{C}$ be a geodesic triangle, for instance, on a circular cylinder $x^{2}+y^{2}=1$ for $z_{1} \leq z \leq z_{2}$ and $F_{C} \equiv A_{0}$ ia the corresponding weighted FermatTorricelli point for given weights $w_{1}, w_{2}$, and $w_{3}$.

By $A_{i}=\left(\cos \varphi_{i}, \sin \varphi_{i}, z_{i}\right)$, we denote the points located on the circular cylinder $x^{2}+y^{2}=1$, by $\left(a_{i j}\right)_{C}$, the length of the geodesic arc $A_{i} A_{j}$, by $\vec{r}_{i j}=$ $\left(\cos t, \sin t, b_{i j} t\right)$, the circular helix from $A_{i}$ to $A_{j}$, by $\left(\alpha_{i j k}\right)_{C}$, the angle formed by $A_{i} A_{j}$ and $A_{j} A_{k}$, by $A_{i p}$, the projection of $A_{i}$ to the circle of the cylinder which passes from $A_{1}=(1,0,0)$, by $\omega_{0}$, the angle $\angle A_{0 p} A_{1} A_{0}$, by $z_{0}$, the linear segment $A_{0} A_{0 p}$, and by $L_{0}$, the linear segment $A_{1} A_{0 p}$ for $i, j, k=0,1,2,3, i \neq j$ and $j \neq k$.

We set $b_{12} \equiv \frac{z_{2}}{\varphi_{2}}$ and $b_{13}=\equiv \frac{z_{3}}{\varphi_{3}}$, where $0<\varphi_{i}<\pi$, for $i, j=1,2,3$ and $i \neq j$. 
We need the following two lemmata proved in [12] and [11] (see also in [12]):

Lemma 1. [12, Theorem 1, p. 173]. The exact location of the weighted Fermat-Torricelli point $A_{0}=A_{0}\left(x_{0}, y_{0}, z_{0}\right)$ of $\triangle\left(A_{1} A_{2} A_{3}\right)_{C}$, composed of three circular helixes on the circular cylinder, is given by the following three equations:

$$
\begin{gathered}
\omega_{0}=\arctan b_{12}+\arccos \left(\frac{1+b_{12} b_{13}}{\sqrt{1+b_{12}^{2}} \sqrt{1+b_{13}^{2}}}\right) \\
-\operatorname{arccot}\left[\left(\sqrt{1-\left(\frac{1+b_{12} b_{13}}{\sqrt{1+b_{12}^{2}} \sqrt{1+b_{13}^{2}}}\right)^{2}}\right.\right. \\
-\frac{1+b_{12} b_{13}}{\sqrt{1+b_{12}^{2}} \sqrt{1+b_{13}^{2}}} \cot \left(\arccos \frac{w_{3}^{2}-w_{1}^{2}-w_{2}^{2}}{2 w_{1} w_{2}}\right) \\
\left.-\frac{\sqrt{1+b_{13}^{2}} \varphi_{3}}{\sqrt{1+b_{12}^{2}} \varphi_{2}} \cot \left(\arccos \frac{w_{2}^{2}-w_{1}^{2}-w_{3}^{2}}{2 w_{1} w_{3}}\right)\right) / \\
\left.-\frac{1+b_{12} b_{13}}{\sqrt{1+b_{12}^{2}} \sqrt{1+b_{13}^{2}}}-\sqrt{1-\left(\frac{\sqrt{1+b_{13}^{2}} \varphi_{3}}{\sqrt{1+b_{12}^{2}} \sqrt{1+b_{13}^{2}}}\right)^{2} \cot (\arccos } \frac{w_{3}^{2}-w_{1}^{2}-w_{2}^{2}}{2 w_{1} w_{2}}\right) \\
\quad \sin \left(\arccos \frac{w_{2}^{2}-w_{1}^{2}-w_{3}^{2}}{2 w_{1} w_{3}}\right) \\
z_{0}=\frac{\sin \left(\arctan b_{13}-\omega_{0}+\arccos \frac{w_{2}^{2}-w_{1}^{2}-w_{3}^{2}}{2 w_{1} w_{3}}\right) \sqrt{1+b_{13}^{2}} \varphi_{3}}{\sin \omega_{0}}
\end{gathered}
$$

and

$$
L_{0}=\frac{\sin \left(\arctan b_{13}-\omega_{0}+\arccos \frac{w_{2}^{2}-w_{1}^{2}-w_{3}^{2}}{2 w_{1} w_{3}}\right) \sqrt{1+b_{13}^{2}} \varphi_{3}}{\sin \left(\arccos \frac{w_{2}^{2}-w_{1}^{2}-w_{3}^{2}}{2 w_{1} w_{3}}\right)} \cos \omega_{0} .
$$

We consider the same points $A_{1}, A_{2}, A_{3}$, and $A_{0}$ on a sphere $S\left(A_{0}, R\right)$ and we denote by $\triangle\left(A_{1} A_{2} A_{3}\right)_{S}$ the geodesic triangle on $S\left(A_{0}, R\right)$, by $\left(a_{i j}\right)_{S}$, the length of the geodesic arc $A_{i} A_{j}$, by $\left(\alpha_{i j k}\right)_{S}$, the angle formed by $A_{i} A_{j}$ and $A_{j} A_{k}$ and $w_{i}^{\prime}$, the weight which corresponds to $A_{i}$ and minimizes the objective function $w_{1}^{\prime}\left(a_{01}\right)_{S}+w_{2}^{\prime}\left(a_{02}\right)_{S}+w_{3}^{\prime}\left(a_{03}\right)_{S}$ for $i, j, k=0,1,2,3$ and $i \neq j \neq k$.

We set

$$
c_{i} \equiv \frac{\sin \left(\kappa\left(a_{j k}\right)_{S}\right)}{\sin \left(\left(\alpha_{j 0 k}\right)_{S}\right)}
$$


for $i, j, k=1,2,3$ and $i \neq j \neq k$, where

$$
\kappa= \begin{cases}\sqrt{K} & \text { if } K=\frac{1}{R^{2}}>0 \\ i \sqrt{-K} & \text { if } K<0\end{cases}
$$

Lemma 2. [13, Theorem 2.4, p. 115]. A finite set of solutions of the weighted Fermat-Torricelli problem on the K-plane(two-dimensional sphere, hyperbolic plane), which yields the global minimum point $A_{0}$ (weighted Fermat-Torricelli point), is given by the following equation with respect to the variable $z=\sin \left(\alpha_{013}\right)_{S}$ :

$$
\begin{gathered}
\frac{c_{3}}{c_{2}}\left( \pm \sin \left(\alpha_{123}\right)_{S} \sqrt{1-\left(\frac{c_{2} z}{c_{1}}\right)^{2}}-\cos \left(\alpha_{123}\right)_{S}\left(\frac{c_{2} z}{c_{1}}\right)\right) \\
=-\frac{c_{3}}{c_{1}} \sin \left(\alpha_{213}\right)_{S} \cos \left(\alpha_{132}\right)_{S} \sqrt{1-z^{2}}+\frac{c_{3}}{c_{1}} \cos \left(\alpha_{213}\right)_{S} \cos \left(\alpha_{132}\right)_{S} z \\
\pm\left(\sin \alpha_{132}\right)_{S} \sqrt{1-\left(\frac{c_{3}}{c_{1}}\right)^{2}\left[-\sin \left(2\left(\alpha_{213}\right)_{S}\right) z \sqrt{1-z^{2}}+\cos \left(2\left(\alpha_{213}\right)_{S}\right) z^{2}+\sin ^{2}\left(\alpha_{213}\right)_{S}\right]} .
\end{gathered}
$$

We recall the inverse weighted Fermat-Torricelli problem on a $C^{2}$ surface in $\mathbb{R}^{3}$ first stated by S. Gueron and $\mathrm{R}$. Tessler in $\mathbb{R}^{2}([2,8,9,10])$ :

Problem 2. [2, p. 449], [8, Problem 3.2, p. 61] [9, Problem 2, p. 52], [10]. Given is a point $A_{0} \in \triangle A_{1} A_{2} A_{3}$ on a $C^{2}$ surface in $\mathbb{R}^{3}$. Does there exist a unique set of positive weights $w_{i}$, normalized by $w_{1}+w_{2}+w_{3}=1$, for which $A_{0}$ minimizes

$$
w_{1}\left(a_{01}\right)_{g}+w_{2}\left(a_{02}\right)_{g}+w_{3}\left(a_{03}\right)_{g},
$$

where $\left(a_{0 i}\right)_{g}$ is the length of the geodesic arc $A_{0} A_{i}$ ?

Lemma 3. [2], [8, Proposition 3.2, Corollary 3.3, p. 61] [9, Proposition 5, p. 52], [10]. The solution of the inverse weighted Fermat-Torricelli problem on a $C^{2}$ surface in $\mathbb{R}^{3}$ is given by

$$
w_{i}=\frac{1}{1+\frac{\sin \alpha_{i 0 j}}{\sin \alpha_{j 0 k}}+\frac{\sin \alpha_{i 0 k}}{\sin \alpha_{j 0 k}}}
$$

for $i, j, k=1,2,3$ and $i \neq j \neq k$.

We assume that $A_{i}=\left(x_{i}, y_{i}, z_{i}\right)$ and $F_{C}=F_{S} \equiv A_{0}=\left(x_{F}, y_{F}, z_{F}\right)$ are located at the intersection of the circular cylinder $C$ and the sphere $S\left(x_{0}, y_{0}, z_{0} ; R\right)$ for $i=1,2,3$. 
Theorem 1. The following equations provide the plasticity of a sphere derived by a circular cylinder with respect to the fixed points $\left\{A_{1} A_{2} A_{3} A_{0}\right\}$ for a different triad of weights $w_{1}, w_{2}, w_{3}$, and $w_{1}^{\prime}, w_{2}^{\prime}, w_{3}^{\prime}$ such that $F_{C}=F_{S} \equiv A_{0}$ :

$$
w_{i}=\frac{1}{1+\frac{\sin \left(\alpha_{i 0 j}\right)_{S}}{\sin \left(\alpha_{j 0 k}\right)_{S}}+\frac{\sin \left(\alpha_{i 0 k}\right)_{S}}{\sin \left(\alpha_{j 0 k}\right)_{S}}},
$$

for $i, j, k=1,2,3$ and $i \neq j \neq k$, the angles $\left(\alpha_{i 0 j}\right)_{S}$ are determined by the equations

$$
\begin{gathered}
x_{0}=-\frac{-d_{3} g_{2} h_{1}+d_{2} g_{3} h_{1}+d_{3} g_{1} h_{2}-d_{1} g_{3}, h_{2}-d_{2} g_{1} h_{3}+d_{1} g_{2} h_{3}}{f_{3} g_{2} h_{1}-f_{2} g_{3} h_{1}-f_{3}, g_{1} h_{2}+f_{1} g_{3} h_{2}+f_{2} g_{1} h_{3}-f_{1} g_{2} h_{3}}, \\
y_{0}=-\frac{d_{3} f_{2} h_{1}-d_{2} f_{3} h_{1}-d_{3} f_{1} h_{2}+d_{1} f_{3} h_{2}+d_{2} f_{1} h_{3}-d_{1} f_{2} h_{3}}{f_{3} g_{2} h_{1}-f_{2} g_{3} h_{1}-f_{3} g_{1} h_{2}+f_{1} g_{3} h_{2}+f_{2} g_{1} h_{3}-f_{1} g_{2} h_{3}}, \\
z_{0}=\frac{d_{3} f_{2} g_{1}-d_{2} f_{3} g_{1}-d_{3} f_{1} g_{2}+d_{1} f_{3} g_{2}+d_{2} f_{1} g_{3}-d_{1} f_{2} g_{3}}{f_{3} g_{2} h_{1}-f_{2} g_{3} h_{1}-f_{3} g_{1} h_{2}+f_{1} g_{3} h_{2}+f_{2} g_{1} h_{3}-f_{1} g_{2} h_{3}}, \\
w_{1}^{\prime}+w_{2}^{\prime}+w_{3}^{\prime}=1
\end{gathered}
$$

where

$$
\begin{aligned}
& f_{1}=\left(x_{i}-x_{F}\right), \\
& g_{1}=\left(y_{i}-y_{F}\right), \\
& h_{1}=\left(z_{i}-z_{F}\right),
\end{aligned}
$$

and

$$
d_{i}=0.5\left[\left(x_{i}-x_{F}\right)\left(x_{i}+x_{F}\right)+\left(y_{i}-y_{F}\right)\left(y_{i}+y_{F}\right)+\left(z_{i}-z_{F}\right)\left(z_{i}+z_{F}\right)\right] .
$$

$\mathrm{P}$ r o o f. Let $\triangle\left(A_{1} A_{2} A_{3}\right)_{C}$ be a geodesic triangle which is composed of three circular helixes on a circular cylinder $x^{2}+y^{2}=1$ for $z_{1} \leq z \leq z_{2}$, and $F_{C}$ be the corresponding weighted Fermat-Torricelli point. By unrolling the cylinder, we get an isometric mapping of $\triangle A_{1} A_{2} A_{3}$ to the Euclidean plane $\mathbb{R}^{2}$. From Lemma 1, we derive the exact location of $F_{C}=\left(x_{F}, y_{F}, z_{F}\right)$.

We construct a sphere $S\left(A_{0}\left(x_{0}, y_{0}, z_{0}\right), R\right)$ which passes from $A_{1}=\left(x_{1}, y_{1}, z_{1}\right)$, $A_{2}=\left(x_{2}, y_{2}, z_{2}\right), A_{3}=\left(x_{3}, y_{3}, z_{3}\right)$ and $F=\left(x_{F}, y_{F}, z_{F}\right)$.

The bisectors of the linear segments $A_{i} F$ pass from $M_{i}=\left(\frac{x_{i}+x_{F}}{2}, \frac{y_{i}+y_{F}}{2}, \frac{z_{i}+z_{F}}{2}\right)$ and intersect at $A_{0}=\left(x_{0}, y_{0}, z_{0}\right)$, such that $\left\|A_{i} A_{0}\right\|=R$, for $i=1,2,3$ (Fig. 1).

Thus, we get

$$
\begin{gathered}
\left(x_{i}-x_{F}\right) x+\left(y_{i}-y_{F}\right) y+\left(z_{i}-z_{F}\right) z \\
=0.5\left[\left(x_{i}-x_{F}\right)\left(x_{i}+x_{F}\right)+\left(y_{i}-y_{F}\right)\left(y_{i}+y_{F}\right)+\left(z_{i}-z_{F}\right)\left(z_{i}+z_{F}\right)\right]
\end{gathered}
$$




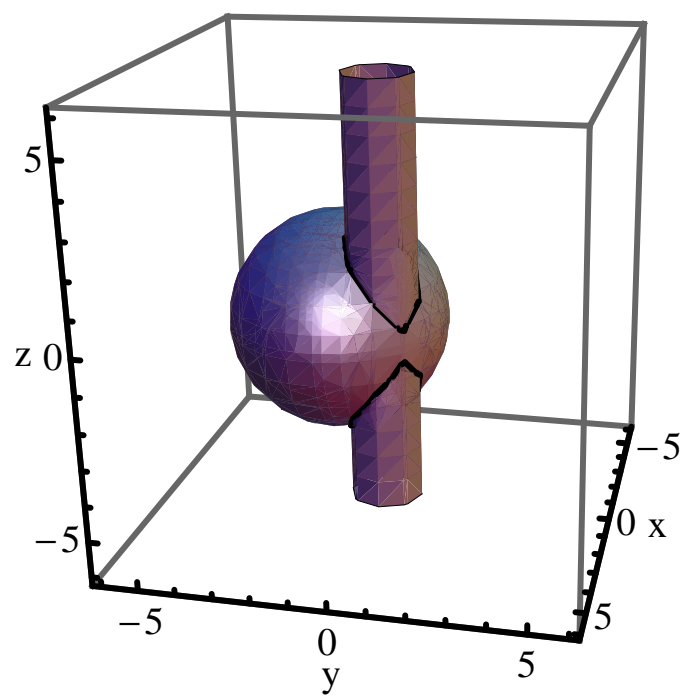

Fig. 1.

for $i=1,2,3$. The intersection of the three planes (2.15) gives (2.7), (2.8), and $(2.9)$.

Thus, we get

$$
\begin{gathered}
R=\frac{1}{\sqrt{K}}=\sqrt{\left(x_{i}-x_{0}\right)^{2}+\left(y_{i}-y_{0}\right)^{2}+\left(z_{i}-z_{0}\right)^{2}}, \\
\cos \theta_{i F}=1-\frac{1}{2}\left(\frac{\left(x_{i}-x_{F}\right)^{2}+\left(y_{i}-y_{F}\right)^{2}+\left(z_{i}-z_{F}\right)^{2}}{R}\right)^{2}, \\
\left(a_{i F}\right)_{S}=R \theta_{i F},
\end{gathered}
$$

and

$$
\left(a_{i j}\right)_{S}=R \theta_{i j}
$$

for $i, j=1,2,3, i \neq j$.

Therefore, the angles $\left(\alpha_{i 0 j}\right)_{S}$ are determined by the spherical cosine law in $\triangle A_{i} A_{j} A_{k}$ :

$$
\left(\alpha_{i 0 j}\right)_{S}=\arccos \frac{\cos \kappa\left(a_{i j}\right)_{S}-\cos \kappa\left(a_{0 i}\right)_{S} \cos \kappa\left(a_{0 j}\right)_{S}}{\sin \kappa\left(a_{0 i}\right)_{S} \sin \kappa\left(a_{0 j}\right)_{S}} .
$$

Then, by applying Lemma 3, we obtain (2.6). 


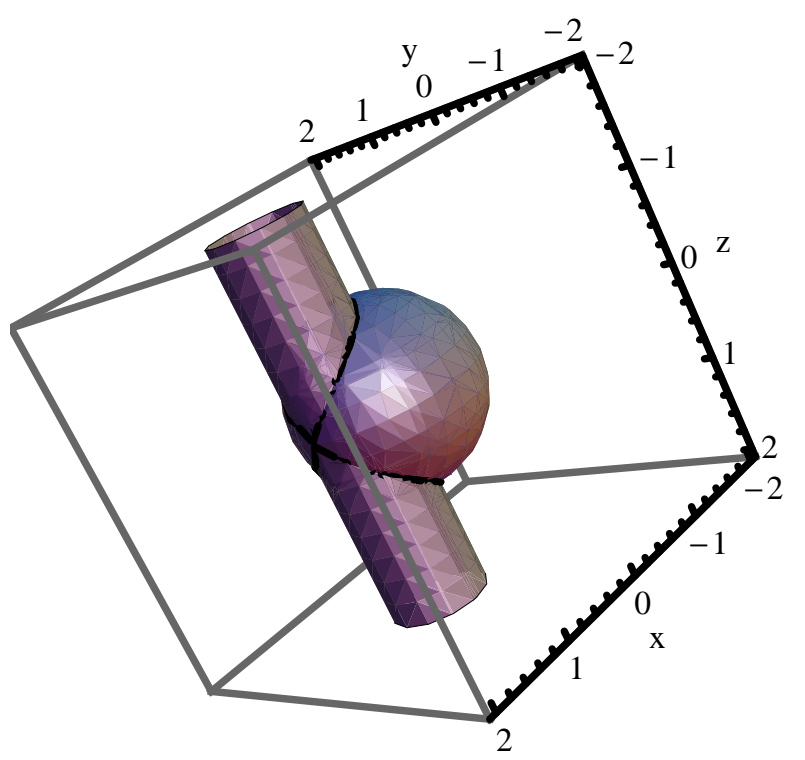

Fig. 2.

$\mathrm{R}$ e $\mathrm{m}$ a r k 1 . We note that there is a particular case where $A_{1}, A_{2}, A_{3}$, and $F$ can be located on a circular cylinder $x^{2}+y^{2}=R x$ with the radius $\frac{R}{2}$ and on a sphere $x^{2}+y^{2}+z^{2}=R^{2}$ with the radius $R$. The intersection of this circular cylinder and the sphere is called a Viviani curve with one point of self-intersection (Fig.2, [6, Example 1.2.4 (a), p. 5]).

We consider the intersection of a circular cylinder $C: x^{2}+y^{2}=1$ for $z_{1} \leq$ $Z \leq z_{2}$ and a circular cone $C o:\left(x-x_{0}\right)^{2}+\left(z-z_{0}\right)^{2}=\left(\frac{r_{1}}{H}\right)^{2}(z-H)^{2}$. By $H$, we denote the height of the circular cylinder and by $r_{1}$, the radius of the circle which corresponds to the basis of the circular cone.

Theorem 2. The following equations provide the plasticity of a circular cone derived by a circular cylinder with respect to the fixed points $\left\{A_{1} A_{2} A_{3} A_{0}\right\}$ for a different triad of weights $w_{1}, w_{2}, w_{3}$, and $w_{1}^{\prime}, w_{2}^{\prime}, w_{3}^{\prime}$ such that $F_{C o}=F_{C} \equiv A_{0}$ :

$$
w_{i}=\frac{1}{1+\frac{\sin \left(\alpha_{i 0 j}\right)_{C o}}{\sin \left(\alpha_{j 0 k}\right)_{C o}}+\frac{\sin \left(\alpha_{i 0 k}\right)_{C o}}{\sin \left(\alpha_{j 0 k}\right)_{C o}}},
$$

and the angles $\left(\alpha_{i 0 j}\right)_{S}$ are determined by the equations

$$
\left(x_{1}-x_{0}\right)^{2}+\left(z_{1}-z_{0}\right)^{2}=\left(\frac{r_{1}}{H}\right)^{2}\left(z_{1}-H\right)^{2},
$$




$$
\begin{aligned}
\left(x_{2}-x_{0}\right)^{2}+\left(z_{2}-z_{0}\right)^{2} & =\left(\frac{r_{1}}{H}\right)^{2}\left(z_{2}-H\right)^{2}, \\
\left(x_{3}-x_{0}\right)^{2}+\left(z_{3}-z_{0}\right)^{2} & =\left(\frac{r_{1}}{H}\right)^{2}\left(z_{3}-H\right)^{2}, \\
\left(x_{F}-x_{0}\right)^{2}+\left(z_{F}-z_{0}\right)^{2} & =\left(\frac{r_{1}}{H}\right)^{2}\left(z_{F}-H\right)^{2},
\end{aligned}
$$

where

$$
w_{1}^{\prime}+w_{2}^{\prime}+w_{3}^{\prime}=1
$$

P r o o f. By considering a fittable circular cone $C o:\left(x-x_{0}\right)^{2}+\left(z-z_{0}\right)^{2}=$ $\left(\frac{r_{1}}{H}\right)^{2}(z-H)^{2}$, which passes from the points $A_{1}, A_{2}, A_{3}$, and $F_{C o} \equiv F_{C}=A_{0}$, we get the system of equations $(2.22),(2.23),(2.24)$, and (2.25) with respect to the four variables $x_{0}, y_{0}, r_{1}$, and $z_{0}=H$, which can give numerically the vertex $A$ of the circular cone.

Then, by unrolling the circular cone $C o$ along $A_{1} A$, we derive an isometric mapping from $C o$ to $\mathbb{R}^{2}$, which determines the angles $\left(\alpha_{i j k}\right)_{0}=\left(\alpha_{i j k}\right)_{C o}$, and obtain (2.21).

Taking into account that $A$ is the vertex of the circular cone, $r_{1}$ is the radius of the circle $c\left(P, r_{1}\right)$ at the basis of the cone, $H$ is the height of the cone, we denote by $\varphi_{0}$ the angle $\angle A_{1} P A_{0 p}$, where $A_{0 p}$ is the point of intersection of $A A_{0}$ and $c\left(P, r_{1}\right)$, and by $x_{00}$, the length of the linear segment $A_{0} A$, and we consider the lemma proved in $([12])$.

Lemma 4. [12, Theorem 2, p. 177-178]. The exact location of the weighted Fermat-Torricelli point $F_{C o}$ of $\triangle A_{1} A_{2} A_{3}$ on $C o$ is given by the following two equations:

$$
x_{00}=\sqrt{\left(1+H^{2}\right)+\left(a_{01}\right)_{g}^{2}-2 \sqrt{1+H^{2}}\left(a_{01}\right)_{g} \cos \left(\alpha_{013}+\angle A_{3} A_{1} A\right)},
$$

where

$$
\begin{gathered}
\alpha_{013}=\operatorname{arccot}\left(\frac{\sin \left(\alpha_{213}\right)-\cos \left(\alpha_{213}\right) \cot \left(\alpha_{102}\right)_{C o}-\frac{\left(a_{13}\right)_{0}}{\left(a_{12}\right)_{0}} \cot \left(\alpha_{103}\right)_{C o}}{-\cos \left(\alpha_{213}\right)-\sin \left(\alpha_{213}\right) \cot \left(\alpha_{102}\right)_{C o}+\frac{\left(a_{13}\right)_{0}}{\left(a_{12}\right)_{0}}}\right), \\
\left(a_{10}\right)_{0}=\frac{\sin \left(\alpha_{013}+\left(\alpha_{103}\right)_{C o}\right)\left(a_{13}\right)_{0}}{\sin \left(\alpha_{103}\right)_{C o}}, \\
\left(\alpha_{i 0 j}\right)_{C o}=\arccos \left(\frac{\left(w_{k}^{\prime}\right)^{2}-\left(w_{i}^{\prime}\right)^{2}-\left(w_{j}^{\prime}\right)^{2}}{2\left(w_{i}^{\prime}\right)\left(w_{j}^{\prime}\right)}\right),
\end{gathered}
$$

for $i, j, k=1,2,3, i \neq j \neq k$, and

$$
\varphi_{0}=\frac{\sqrt{1+H^{2}}}{r_{1}} \angle A_{1} A A_{0}\left(x_{00},\left(a_{10}\right)_{g}\right) .
$$


$\mathrm{R}$ e $\mathrm{m}$ a $\mathrm{r} \mathrm{k} 2$. For given $x_{00}, \alpha_{013}$ and $\left(a_{10}\right)_{0}$, the system of two nonlinear equations (2.27) and (2.28) gives numerically $w_{1}^{\prime}, w_{2}^{\prime}$, taking into account that $w_{3}^{\prime}=1-w_{1}^{\prime}-w_{2}^{\prime}$.

E x a m p l e 1 . Given are $A_{1}=(\cos 0, \sin 0,0), A_{2}=\left(\cos \frac{\pi}{3}, \sin \frac{\pi}{3}, 0.8\right)$, $A_{3}=\left(\cos \frac{\pi}{6}, \sin \frac{\pi}{6}, 2\right)$, on the circular cylinder $x^{2}+y^{2}=1$.

The isometric mapping of the circular cylinder to $\mathbb{R}^{2}$ induced by $(\varphi, z)$ yields the points $A_{1}^{\prime}=(0,0), A_{2}^{\prime}=\left(\frac{\pi}{3}, 0.8\right), A_{3}^{\prime}=\left(\frac{\pi}{6}, 2\right)$. Thus, the corresponding Fermat-Torricelli point of $\triangle A_{1}^{\prime} A_{2}^{\prime} A_{3}^{\prime} F^{\prime}=(0.8404027,0.8536775)$ gives $F_{C}=$ $(0.667163,0.744912,0.8536775)$ for $w_{1}=w_{2}=w_{3}=\frac{1}{3}$.

Given $A_{1}, A_{2}, A_{3}, F_{C}$, we calculate the center of the fittable sphere $x_{0}, y_{0}, z_{0}, R$ from the equations (2.7)-(2.14): $x_{0}=-1.31848, y_{0}=-1.60442, z_{0}=1.31278$, $R=3.11013$.

From (2.17), (2.18), (2.19), (2.20), and (2.6), we derive that

$$
\begin{aligned}
& \left(\alpha_{102}\right)_{S}=1.99478 \mathrm{rad}, \\
& \left(\alpha_{203}\right)_{S}=2.10237 \mathrm{rad}, \\
& \left(\alpha_{103}\right)_{S}=2.18604 \mathrm{rad},
\end{aligned}
$$

which give

$$
\begin{aligned}
w_{1}^{\prime} & =0.33281, \\
w_{2}^{\prime} & =0.315291
\end{aligned}
$$

and

$$
w_{3}^{\prime}=0.3519
$$

such that

$$
w_{1}^{\prime}+w_{2}^{\prime}+w_{3}^{\prime}=1
$$

As a future work, we consider the following problem that may provide some perspectives on the plasticity of geodesic triangles on some $C^{2}$ complete surfaces in $\mathbb{R}^{3}$ :

Problem 3. Suppose that $F$ is the corresponding Fermat-Torricelli point of a geodesic triangle $\triangle A_{1} A_{2} A_{3}$ on a $C^{2}$ complete surface $M$ with positive weights $w_{i}$ such that

$$
w_{1}+w_{2}+w_{3}=1
$$

Find a fittable Alexandrov surface $M^{\prime}$ of a bounded curvature which passes from $A_{1}, A_{2} A_{3}$ and $F$ such that $F$ is the corresponding Fermat-Torricelli point of $\triangle A_{1} A_{2} A_{3}$ on $M^{\prime}$, with positive weights $w_{i}^{\prime}$, satisfying the equations

$$
w_{1}^{\prime}+w_{2}^{\prime}+w_{3}^{\prime}=1
$$

and $w_{1}=w_{1}^{\prime}$ or $w_{1}=w_{1}^{\prime}$ and $w_{2}=w_{2}^{\prime}$. 
R e m a r k 3 . If the points $A_{1}, A_{2}, A_{3}$ are not fixed and belong to the surface of a circular cylinder, then there is an isometric mapping which is deduced by unrolling the circular cylinder by the line (generator of cylinder) which passes from the weighted Fermat-Torricelli point $A_{0}$ of $\triangle\left(\triangle A_{1} A_{2} A_{3}\right)_{C}$ and the corresponding weighted Fermat-Torricelli point of $\triangle\left(\triangle A_{1} A_{2} A_{3}\right)_{P}$ on the Euclidean plane coincides with $A_{0}$, which yields $w_{1}=w_{1}^{\prime}$ and $w_{2}=w_{2}^{\prime}$.

We are interested in the derivation of non-isometric mappings of fittable surfaces which solve Problem 3, which could also lead to a new way of creating two-dimensional fittable hyperbolic spaces (Plasticity of hyperbolic spaces).

Finally, we note that Problems 1 and 3 may provide an alternative characterization of a Wald curvature ([7]) by placing geometric properties of the weighted Fermat-Torricelli problem for geodesic triangles into Wald's nonlinear quad.

\section{References}

[1] V. Boltyanski, H. Martini, and V. Soltan, Geometric Methods and Optimization Problems. Kluwer, Dordrecht-Boston-London, 1999.

[2] S. Gueron and R. Tessler, The Fermat-Steiner Problem. - Amer. Math. Monthly 109 (2002), 443-451.

[3] A.O. Ivanov and A.A. Tuzhilin, Geometry of Minimal Nets and the One-dimensional Plateau Problem. - Russian Math. Surveys 47 (1992), No. 2, 59-131.

[4] A.O. Ivanov and A.A. Tuzhilin, What Spaces Permit Fermat Points Construction and Melzak Algorithm.

http://ftp.uniyar.ac.ru/sites/default/files/papers/problems/

[5] S. Naya and N. Innami, A Comparison Theorem for Steiner Minimum Trees in Surfaces with Curvature Bounded Below. - Tohoku Math. J. 65 (2013), No. 1, $131-157$.

[6] V.A. Toponogov, Differential Geometry of Curves and Surfaces. Birkhäuser, 2005.

[7] A. Wald, Begründung einer Koordinatenlosen Differentialgeometrie der Flachen. Ergebnisse eines Mathematischen Kolloquiums 7 (1935), 24-46.

[8] A.N. Zachos and G. Zouzoulas, The Weighted Fermat-Torricelli Problem and an "Inverse" Problem. - J. Convex Anal. 15 (2008), No. 1, 55-62.

[9] A. Zachos and A. Cotsiolis, The Weighted Fermat-Torricelli Problem on a Surface and an "Inverse" Problem. - J. Math. Anal. Appl. 373 (2011), No. 1, 44-58.

[10] A. Cotsiolis and A. Zachos, Corrigendum to "The Weighted Fermat-Torricelli Problem on a Surface and an "Inverse" Problem". - J. Math. Anal. Appl. 376 (2011), No. 2. 
[11] A. Zachos, Location of the Weighted Fermat-Torricelli Point on the $K$-plane. Analysis (Munich) 33 (2013), No. 3, 243-249.

[12] A. Zachos, Exact Location of the Weighted Fermat-Torricelli Point on Flat Surfaces of Revolution. — Results Math. 65 (2014), No. 1-2, 167-179.

[13] A. Zachos, Location of the Weighted Fermat-Torricelli Point on the $K$-plane. Part II. - Analysis (Munich) 34 (2014), No. 1, 111-120. 